\title{
Renormalization scale-fixing for complex scattering amplitudes
}

\author{
S.J. Brodsky ${ }^{1, a}$, F.J. Llanes-Estrada ${ }^{2, b}$ \\ 1 Stanford Linear Accelerator Center, Stanford University, Stanford, California, 94309, USA \\ ${ }^{2}$ Depto. Física Teórica I, Fac. Cc. Físicas, Universidad Complutense de Madrid, 28040 Madrid, Spain
}

Received: 21 December 2005 / Revised version: 9 February 2006 /

Published online: 12 April 2006 - (C) Springer-Verlag / Società Italiana di Fisica 2006

\begin{abstract}
We show how to fix the renormalization scale for hard-scattering exclusive processes such as deeply virtual meson electroproduction by applying the BLM prescription to the imaginary part of the scattering amplitude and employing a fixed- $t$ dispersion relation to obtain the scale-fixed real part. In this way, we resolve the ambiguity in BLM renormalization scale-setting for complex scattering amplitudes. We illustrate this by computing the $H$ generalized parton distribution at leading twist in an analytic quark-diquark model for the parton-proton scattering amplitude that can incorporate Regge exchange contributions characteristic of the deep inelastic structure functions.
\end{abstract}

PACS. 11.55.Fv; 11.10.Gh

\section{BLM renormalization scale setting}

A typical QCD amplitude for an exclusive process can be calculated as a power series in the strong coupling constant

$$
A=A^{(0)}+A^{(1)} \alpha_{\mathrm{s}}\left(\mu^{2}\right)+A^{(2)} \alpha_{\mathrm{s}}^{2}\left(\mu^{2}\right)+\ldots
$$

The renormalization scale $\mu$ of the running coupling in such processes can be set systematically in QCD without ambiguity at each order in perturbation theory using the Brodsky-Lepage-Mackenzie (BLM) method [1-3].

The BLM scale is derived order-by-order by incorporating the non-conformal terms associated with the $\beta$ function into the argument of the running coupling. This can be done systematically using the skeleton expansion $[4,5]$. The scale determined by the BLM method is consistent with (a) the transitivity and other properties of the renormalization group [6], (b) the renormalization group principle that relations between observables must be independent of the choice of intermediate renormalization scheme $[7,8]$, and (c) the location of the analytic cut structure of amplitudes at each flavor threshold. The non-conformal terms involving the $\mathrm{QCD} \beta$ function are all absorbed by the scale choice. The coefficients of the perturbative series remaining after BLM-scale-setting are thus the same as those of a conformally invariant theory with $\beta=0$. In practice, one can often simply use the flavor dependence of the series to tag the non-conformal $\beta$-dependence in perturbation theory; i.e., the BLM procedure resums the terms involving $n_{f}$ associated with the running of the QCD coupling.

\footnotetext{
a e-mail: sjbth@slac.stanford.edu

b e-mail: fllanes@fis.ucm.es
}

Non-Abelian gauge theory based on $S U\left(N_{C}\right)$ symmetry becomes an Abelian QED-like theory in the limit $N_{C} \rightarrow 0$, while keeping $\alpha=C_{F} \alpha_{\mathrm{s}}$ and $n_{\ell}=n_{\text {eff }} / 2 C_{F}$ fixed [9]. Here $C_{F}=\left(N_{C}^{2}-1\right) / 2 N_{C}$. The BLM scale reduces properly to the standard QED scale in this analytic limit. For example, consider the vacuum polarization lepton-loop correction to $\mathrm{e}^{+} \mathrm{e}^{-} \rightarrow \mathrm{e}^{+} \mathrm{e}^{-}$in QED. The amplitude must be proportional to $\alpha(s)$, since this gives the correct cut of the forward amplitude at the lepton pair threshold $s=4 m_{\ell}^{2}$. Thus the renormalization scale $\mu_{R}^{2}=s$ is exact and unambiguous in the conventional QED Goldberger-Low scheme [10]. If one chooses any other scale $\mu_{R}^{2} \neq s$, the scale $\mu_{R}^{2}=s$ will be restored when one sums all bubble graphs. The BLM procedure is thus consistent with the Abelian limit and the proper cut structure of amplitudes.

\section{Difficulties in using the mean value theorem to set the BLM scale}

The BLM scale at leading order has a simple physical interpretation: it is identical to the photon virtuality in QED applications and the mean gluon virtuality in QCD when one uses physical schemes that generalize the QED scheme, such as the pinch scheme $[2,11]$ and the $\alpha_{\mathrm{V}}[5,12]$ scheme defined from the QCD static potential. The number of flavors active in virtual corrections to a given process is evident from the BLM scale choice: the BLM method sets the renormalization scale so that the flavor number is changed properly in any renormalization scheme, including the $\overline{\mathrm{MS}}$ scheme $[2,13]$.

In effect, the BLM prescription identifies the renormalization scale and the gluon virtuality by eliminat- 
ing the dependence on the number of flavors from the $O\left(\alpha_{\mathrm{s}}\right)$ (expanding $\alpha_{\mathrm{s}}$ itself) and $O\left(\alpha_{\mathrm{s}}^{2}\right)$ non-conformal terms in the perturbative amplitude. Typically, a QCD amplitude involves an integral over the momentum running through the gluon propagator. Therefore, the argument of the coupling, if taken to be the momentum flowing through the gluon, varies through the integration phase space. A mean $\bar{Q}^{2}$ can be extracted from the integral if the mean value theorem (MVT) of integral calculus can be applied. The essential requirement for the applicability of the MVT is that the function being evaluated at its mean value has to be continuous through the interval and the weight function has to be Riemannintegrable; this includes weight functions bounded and continuous in the range of integration. However, these necessary conditions are not a property of a principal value integrand associated with a pole that appears, for example, in Compton scattering and deeply virtual meson electroproduction (DVME); as we show explicitly in Sect. 2.1 below, the MVT does not apply for these amplitudes.

It was recently pointed out in [14] that the MVT prescription for determining the BLM procedure fails for amplitudes that are genuinely complex, that is, display non-vanishing real and imaginary parts. The authors then argue that there is no guarantee that the BLM prescription will yield the same answer for both parts of the amplitude. Worse, in the particular example that they study, $\rho$ and $\widehat{\pi}_{1}$ electroproduction, the scale obtained from the real part becomes discontinuous (zero to infinity) at a particular kinematical point due to a divergence in some of the intermediate functions.

In this paper, we note that in a quantum field theory, the real and imaginary parts of a scattering amplitude are not independent, but are constrained due to causality, locality, and Lorentz invariance. This manifests itself in the form of the dispersion relations traditionally used in meson photoproduction $[15,16]$ to link both parts of the amplitude. By examining a simple example in the next subsection, we show that the correct prescription for finding the BLM renormalization scales is to first fix the scale in the imaginary part, and then subsequently, the real part can be obtained by means of a dispersion relation. The BLM scales for the real and imaginary parts are thus not generally equal. We perform an explicit calculation for longitudinally polarized vector meson electroproduction at non-zero skewness in Sect. 2.2.

\subsection{Example of the failure of the mean value theorem}

To illustrate how the MVT can fail for an amplitude that contains a pole, consider the following simple integral:

$$
I[f](y)=\int_{-1}^{1} f(x) \frac{\mathrm{d} x}{x-y+\mathrm{i} \epsilon}
$$

which is a functional of $f$ and a function of $y$. The MVT would state that there would exist a certain $\bar{x}(y)$ in the in- terval $(-1,1)$ such that

$$
I[f](y)=f(\bar{x}(y)) I[1](y) .
$$

For $f=$ constant the theorem holds trivially. However, now consider a linear function $f(x)=A x+B$, with $A, B$ arbitrary real constants. Substituting Cauchy's principal value for distributions (the imaginary part arising for $y \in$ $(-1,1))$

$$
\frac{1}{x-y+\mathrm{i} \epsilon}=P V\left[\frac{1}{x-y}\right]-\mathrm{i} \pi \delta(x-y)
$$

one easily finds

$$
\begin{aligned}
I[f](y) & =2 A+(B+A y)\left(\log \left(\frac{1-y}{1+y}\right)-\mathrm{i} \pi\right) \\
& =2 A+f(y) I[1](y) .
\end{aligned}
$$

The MVT thus holds for the imaginary part, but it fails for the real part by the term $2 A$. Even in the instance where $f$ could be chosen so that a certain $\bar{x}$ would satisfy the theorem for the real part, there would be no reason for $\bar{x}$ to be the same for the imaginary part.

In this example, the function $I[f](y)$ is cut in the complex $y$ plane in the interval $[-1,1]$ of the real axis. The function satisfies an unsubtracted dispersion relation:

$$
\begin{aligned}
I[f](y) & =\frac{1}{2 \pi \mathrm{i}} \int_{\text {cut }} \frac{\mathrm{d} y^{\prime} I[f]\left(y^{\prime}\right)}{y-y^{\prime}-\mathrm{i} \epsilon} \\
\operatorname{Re} I[f](y) & =\frac{1}{2} \int \mathrm{PV}\left[\frac{1}{y-y^{\prime}}\right] \mathrm{d} y^{\prime} \operatorname{Im} I[f]\left(y^{\prime}\right),
\end{aligned}
$$

and thus the discontinuity across the cut is sufficient to reconstruct the whole function. In this trivial example the discontinuity is

$$
I[f](y+\mathrm{i} \epsilon)-I[f](y-\mathrm{i} \epsilon)=-2 \pi \mathrm{i} f(y)
$$

and the mean value "scale" for the imaginary part is $\bar{x}(y)=$ $y$. Once this $\bar{x}$ has been chosen, the full amplitude is easily reconstructed from the dispersion relation.

This procedure is not restricted to linear functions. For an arbitrary polynomial one can expand around $y$,

$$
f(x)=f(y)+\sum_{n=1}^{N} \frac{f^{(n)}(y)}{n !}(x-y)^{n} .
$$

We have now

$I[f](y)=f(y) I[1](y)+\sum_{n=1}^{N} \frac{f^{(n)}(y)}{n \cdot n !}\left((1-y)^{n}-(-1-y)^{n}\right)$.

Again, the second term is "unexpected" and a naive application of the mean value theorem to the real part fails. This additional term is a sum of binomials of increasing degree, which in the limit $N \rightarrow \infty$ can be used to construct any entire function. Since it is analytic, it provides no contribution to the discontinuity across the cut, and the dispersion relation in (6) still holds. 


\subsection{Meson electroproduction}

We now turn to the problem of determining the renormalization scale for vector meson electroproduction $\gamma^{*} p \rightarrow$ $V^{0} p^{\prime}$, the critical example studied in [14]. We will employ the same kinematics, frame choice, and conventions as in [17]. It will be useful to note here that in the asymmetric frame employed, the skewness variable plays the role of Bjorken's $x$, and

$$
\zeta=\frac{Q^{2}}{Q^{2}+s-M_{N}^{2}} ; Q^{2}=s+t+u-2 M_{N}^{2} .
$$

The skewness is in the interval

$$
\zeta \in\left[0, \frac{-t}{2 M^{2}}\left(\sqrt{1+\frac{4 M^{2}}{(-t)}}-1\right)\right] .
$$

As a specific example of meson electroproduction, we consider the production of a $\rho^{0}$ meson by a longitudinally polarized virtual photon $\gamma_{\mathrm{L}}^{*}$ with large virtuality $Q^{2}=$ $-q^{2}[18,19]$. The relation of the differential cross section to the generalized parton distributions is well-known [20]

$$
\frac{\mathrm{d} \sigma_{L}}{\mathrm{~d} t}=\frac{1}{16 \pi\left(s-M_{N}^{2}\right) \Lambda^{1 / 2}\left(s,-Q^{2}, M_{N}^{2}\right)} \frac{1}{2} \sum_{\lambda \lambda^{\prime}}\left|M^{L}\right|^{2}
$$

where $\Lambda^{1 / 2}$ is Källen's function,

$$
\Lambda^{1 / 2}(a, b, c)=\sqrt{a^{2}+b^{2}+c^{2}-2(a b+a c+b c)},
$$

and the amplitude to leading order in $\alpha_{\mathrm{s}}$ and $Q$, ignoring the $E$ generalized parton distribution (GPD), is

$$
\begin{aligned}
\mathrm{i} M_{\rho^{0}}^{L}= & -\mathrm{ie} \frac{4}{9} \frac{1}{Q} 4 \pi \alpha_{\mathrm{s}}\left(\mu^{2}\right) \bar{U}_{\lambda}(P) \gamma^{+} U_{\lambda^{\prime}}\left(P^{\prime}\right) \\
& \times \int_{0}^{1} \mathrm{~d} z \Phi_{\rho}(z) \frac{1}{4 z P^{+}} \int_{\zeta-1}^{1} \mathrm{~d} x H_{\rho_{L}^{0}}^{P}(x, \zeta, t) \\
& \times\left[\frac{1}{x-\zeta-\mathrm{i} \epsilon}+\frac{1}{x+\mathrm{i} \epsilon}\right] .
\end{aligned}
$$

We use a model for the GPD $H$ in this expression specified below in Sect. 3. Much useful information on GPD's has been collected in [21]. Notice that at large $s$ one should also take into account the double gluon exchange between the nucleon and the photon projectile (exiting the reaction as a meson). Substituting now

$$
\bar{U}_{\lambda}(P) \gamma^{+} U_{\lambda^{\prime}}\left(P^{\prime}\right)=2 P^{+} \delta_{\lambda \lambda^{\prime}} \frac{\sqrt{1-\zeta}}{1-\zeta / 2}
$$

and employing the asymptotic value for the vector meson distribution amplitude [22]

$$
\Phi_{\rho}(z)=6 z(1-z) f_{\rho}
$$

with $f_{\rho}=0.216 \mathrm{GeV}$, we obtain

$$
\begin{aligned}
\operatorname{Im} M_{\rho^{0}}^{L}= & \frac{-\mathrm{e} \sqrt{1-\zeta}}{1-\zeta / 2} \frac{4}{9} \frac{3 f_{\rho}}{2} 4 \pi^{2} \alpha_{\mathrm{s}}\left(\mu_{\mathrm{BLM}}^{2}\right) \delta_{\lambda \lambda^{\prime}} \frac{1}{Q} \\
& \times\left[H_{\rho_{L}^{0}}^{P}(0, \zeta, t)-H_{\rho_{L}^{0}}^{P}(\zeta, \zeta, t)\right] .
\end{aligned}
$$

Once the BLM scale is fixed, as in (22) below, the real part of the amplitude to leading order can be accessed by a dispersion relation

$$
\operatorname{Re} M_{\rho^{0}}^{L}=\frac{1}{\pi} \int_{\left(M_{N}+m_{\rho}\right)^{2}}^{\infty} \operatorname{Im} M_{\rho^{0}}^{L} \mathrm{P} V\left[\frac{1}{s^{\prime}-s}\right] \mathrm{d} s^{\prime} .
$$

A few remarks are in order: the $\frac{1}{Q}$ factor in (17) makes the dispersion integral in (18) convergent at fixed $\zeta$ (the Bjorken limit). This is the kinematical case traditionally considered in meson electroproduction [20]. Further, the threshold for the integral is irrelevant if one considers the large $Q$ behavior of $M_{\rho^{0}}^{L}$. The use of a dispersion relation at fixed positive skewness $\zeta$ induces a left cut in the complex $s$ plane due to the photon becoming time-like there. This and the usual $t$-channel left cut are consistently neglected in (18) as they are $1 / s\left(1 / Q^{2}\right.$ at fixed $\left.\zeta\right)$ suppressed. Finally and most importantly, the $1 / Q$ factor in (18) relative to the corresponding Compton amplitude makes the Compton cross section fall much slower in $Q$ than its meson electroproduction counterpart. This is due to the extra gluon necessary to construct the LO distribution amplitude of the emitted meson and is a striking manifestation of the $J=0$ fixed pole that appears in the Compton amplitude due to the quasi-local coupling of two currents to the propagating quark [23].

Let us now turn to setting the BLM renormalization scale for meson electroproduction. We shall disregard the $\mu$ evolution of $H$ as an irrelevant complication for our application. Since the leading order is $\alpha_{\mathrm{s}}$, and the NLO amplitude is available, we can follow [14]. One identifies the NLO contributions from the vacuum polarization (which can be picked up from its dependence on $N_{f}$ ) and imposes the BLM scale-fixing condition on the imaginary part of the amplitude

$$
\operatorname{Im} \int_{0}^{1} \mathrm{~d} z \Phi_{\rho}(z) \int_{\zeta-1}^{1} \frac{\mathrm{d} x}{1-\zeta / 2} H_{\rho_{L}^{0}}^{P}(x, \zeta, t) T=0,
$$

with the $\overline{\mathrm{MS}}$ expression

$$
\begin{aligned}
T= & \frac{1}{z(x-\mathrm{i} \epsilon)}\left[\frac{5}{3}-\log \left(\frac{z x}{\zeta}\right)-\log \left(\frac{Q^{2}}{\mu^{2}}\right)\right] \\
& +\frac{1}{(1-z)} \frac{1}{x-\zeta+\mathrm{i} \epsilon}\left[\frac{5}{3}-\log \left(\frac{(1-z)(x-\zeta)}{\zeta}\right)\right. \\
& \left.-\log \left(\frac{Q^{2}}{\mu^{2}}\right)\right] .
\end{aligned}
$$

Following [24], we first notice that the $z$ integrals are trivial when employing the meson distribution amplitude in (16),

$$
\begin{aligned}
\int_{0}^{1} \mathrm{~d} z \Phi_{\rho}(z) \frac{\log z}{z} & =-\frac{9 f_{\rho}}{2}, \\
\int_{0}^{1} \mathrm{~d} z \Phi_{\rho}(z) \frac{\log (1-z)}{1-z} & =-\frac{9 f_{\rho}}{2} \\
\int_{0}^{1} \mathrm{~d} z \Phi_{\rho}(z) \frac{1}{z} & =3 f_{\rho}, \\
\int_{0}^{1} \mathrm{~d} z \Phi_{\rho}(z) \frac{1}{1-z} & =3 f_{\rho},
\end{aligned}
$$


which allows us to find simpler expressions than those in [14]. Solving for $\mu^{2}$ yields the BLM scale

$$
\mu^{2}=Q^{2} \mathrm{e}^{-A_{1} / A_{2}}
$$

in the $\overline{\mathrm{MS}}$ scheme, where

$$
\begin{aligned}
A_{2}= & \pi[H(0, \zeta, t)-H(\zeta, \zeta, t)] \\
A_{1}= & \left(\frac{19}{6}+\log \zeta\right) A_{2}-\pi(-H(\zeta, \zeta, t) \log (1-\zeta) \\
& +\int_{\zeta-1}^{0} \mathrm{~d} x \frac{H(x, \zeta, t)-H(0, \zeta, t)}{x} \\
& \left.-\int_{\zeta}^{1} \mathrm{~d} x \frac{H(x, \zeta, t)-H(\zeta, \zeta, t)}{x-\zeta}\right) .
\end{aligned}
$$

We also note that if (17) is $N_{f}$-independent, then (18) is also $N_{f}$-independent, and the goal of the BLM scale fixing procedure is achieved simultaneously for the real and imaginary parts of the amplitude thanks to the dispersion relation.

We thus have a totally consistent scale setting procedure at the level of the amplitude. Choosing to fix the BLM scale at the level of the total cross section is, therefore, an unnecessary complication. The reader may observe that since both $A_{1}$ and $A_{2}$ are linear in $H$, the scale in (22) is independent of the absolute normalization of $H$. This would change if the $E$ or other GPD's were included in the analysis. The same observation applies to the normalization of the $\rho$ meson distribution amplitude; i.e., $\mu$ is independent of $f_{\rho}$ in our approximation. Thus the BLM renormalization scales are effectively independent of the flavor of the meson produced.

\section{Covariant quark-diquark model for the $H$ generalized parton distribution}

To complete the evaluation of this specific example, we need a physically inspired model for $H$. We recall the analytic model for virtual Compton scattering introduced in [25], which is the gauge invariant leading twist extension of the "covariant parton model" for structure functions given in [26]. This model can also incorporate not only Reggeon exchange in the $t$ channel, but also the $q^{2}$ independent and $t$-independent $J=0$ fixed-pole contribution from the local coupling of the two photons to the struck quark. The $J=\alpha_{R}=1 / 2$ Regge contributions to the structure functions are evidenced by the $\sqrt{x}$ behavior of the non-singlet structure function $F_{2}^{p}\left(x, Q^{2}\right)-F_{2}^{n}\left(x, Q^{2}\right)$ at small $x$. Since this model has not been widely used in the past few years, we find it worthwhile to readdress it in the modern context as a contribution to the current discussion on GPD's.

The central ansatz of the covariant parton model [26] is to construct a model of the quark-parton scattering amplitude, a vertex with two quark legs of momentum $k, k^{\prime}$ and spin indices $i, i^{\prime}$ and two proton legs with momentum $p, p^{\prime}$ and helicities $\lambda, \lambda^{\prime}$. Since the parton legs are not onshell, this amplitude is a function of four different Lorentz scalars that can be chosen as the three Mandelstam invariants $s_{p p}=\left(p+k^{\prime}\right)^{2}, t_{p p}=\left(k^{\prime}-k\right)^{2}=\left(p^{\prime}-p\right)^{2}, u_{p p}=(p-$ $k)^{2}$ and $k^{2}$. The squared momentum of the returning parton can be expressed as ${k^{\prime}}^{2}=s_{p p}+t_{p p}+u_{p p}-2 M_{N}^{2}-k^{2}$. We will denote this amplitude by

$$
T_{\lambda \lambda^{\prime} ; \mathrm{i}^{\prime} \mathrm{i}}\left[s_{p p}, t_{p p}, u_{p p}, k^{2}\right] .
$$

In terms of the DVME scattering kinematical variables $x$, $\boldsymbol{\Delta}_{\perp}, \zeta, t$, and the integration variable $k$, we have the following expressions for the parton-proton scattering matrix Lorentz invariants:

$$
\begin{aligned}
s_{p p}= & (1+x-\zeta) P^{+}\left(k^{-}+\frac{M^{2}+\boldsymbol{\Delta}_{\perp}^{2}}{(1-\zeta) P^{+}}\right)-\left(\mathbf{k}_{\perp}-\boldsymbol{\Delta}_{\perp}\right)^{2} \\
u_{p p}= & \left(P^{+} k^{-}-M_{N}^{2}\right)(x-1)-\mathbf{k}_{\perp}^{2} \\
t_{p p}= & t \\
k^{2}= & x P^{+} k^{-}-\mathbf{k}_{\perp}^{2} \\
k^{\prime 2}= & t+M_{N}^{2}(1-x)+P^{+} k^{-}(x-\zeta)+\left(M_{N}^{2}+\boldsymbol{\Delta}_{\perp}^{2}\right) \\
& \times\left(1+\frac{x}{1-\zeta}\right)-\left(\mathbf{k}_{\perp}-\boldsymbol{\Delta}_{\perp}\right)^{2}
\end{aligned}
$$

(hereafter the subindex ${ }_{p p}$ can be dropped without confusion). To connect with the modern formulation of deeply virtual Compton scattering in terms of GPD's, let us note [21] that

$$
\begin{aligned}
& \frac{1}{2 P^{+}} \bar{U}\left(P^{\prime}\right)_{\lambda^{\prime}}\left[H(x, \zeta, t) \gamma^{+}-E(x, \zeta, t) \frac{\mathrm{i} \sigma^{+\alpha} \Delta_{\alpha}}{2 M_{N}}\right] U(P)_{\lambda} \\
& =\left.\int \frac{\mathrm{d} y^{-}}{8 \pi} \mathrm{e}^{\mathrm{i} x P^{+} y^{-} / 2}\left\langle P^{\prime}, \lambda^{\prime}\left|\bar{\Psi}(0) \gamma^{+} \Psi(y)\right| P, \lambda\right\rangle\right|_{y_{+}=0, y_{\perp}=0} \\
& =\frac{1}{2} \int \frac{\mathrm{d}^{4} k}{(2 \pi)^{4}} \delta\left(x P^{+}-k^{+}\right) \int \frac{\mathrm{d}^{4} k^{\prime}}{(2 \pi)^{4}} \delta\left((x-\zeta) P^{+}-k^{\prime}\right) \\
& \quad \times \gamma_{\mathrm{ii}^{\prime}}^{+}(2 \pi)^{4} \delta^{(4)}\left(p+k^{\prime}-p^{\prime}+k\right) T_{\lambda \lambda^{\prime} ; \mathrm{i}^{\prime} \mathrm{i}}\left[s, t, u, k^{2}\right],
\end{aligned}
$$

where the Fourier modes of the fermion field are

$$
\widehat{\Psi}(k)=\int \mathrm{d}^{4} y \mathrm{e}^{\mathrm{i} k y} \Psi(y) \delta^{(2)}\left(\mathbf{y}_{\perp}\right) \delta\left(y^{+}\right) .
$$

This allows one to construct the leading twist handbag contributions to the GPD's given a model for the partonproton scattering amplitude, by integrating over parton transverse and $(-)$ momentum, and contracting the spins with a $\gamma^{+}$matrix in the parton Dirac space.

The simplest model for the parton-proton amplitude is a tree-level diagram based on perturbation theory with a proton-quark-diquark vertex (see Fig. 1). Since we are only interested in leading twist effects in the electroproduction amplitude, we will ignore the axial vector diquark and the spin dependence of the vertex. The proton spin in this model, with an $s$-wave proton-quark-diquark vertex, is carried by the struck quark, so that

$$
T_{\lambda \lambda^{\prime} ; \mathrm{i}^{\prime} \mathrm{i}}\left[s, t, u, k^{2}\right]=T\left[s, t, u, k^{2}\right] \delta_{\lambda \lambda^{\prime}} \delta_{\mathrm{ii}^{\prime}}
$$



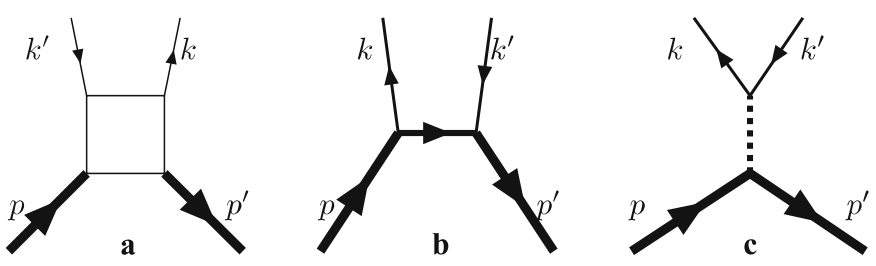

Fig. 1. a Quark-proton scattering matrix. b Quark-proton $u$ channel diquark exchange. c Quark-proton $t$-channel pomeron/ Reggeon exchange. The need for modeling Regge trajectories is avoided by requiring $t \ll-1 \mathrm{GeV}^{2}$, where $\alpha(t) \rightarrow-1[27]$ and $u^{\alpha(t)} \propto \frac{1}{u}$, which can be reabsorbed in the quark-diquark model

for the $H$ GPD part, which yields the oversimplified, yet practical equation

$$
\frac{1}{2 P^{+}} H(x, \zeta, t)=\frac{1}{2} \int \frac{\mathrm{d}^{4} k}{(2 \pi)^{4}} \delta\left(P^{+} x-k^{+}\right) T\left[s, t, u, k^{2}\right] .
$$

The amplitude in covariant perturbation theory is

$$
\mathrm{i} T\left(s, t, u, k^{2}, k^{\prime 2}\right)=\left(\mathrm{i} g\left(k^{2}\right)\right) \frac{\mathrm{i}}{(p-k)^{2}-\lambda^{2}+\mathrm{i} \epsilon}\left(\mathrm{i} g\left(k^{\prime 2}\right)\right) .
$$

Here $\lambda$ plays the role of a scalar diquark mass and $g$ is the amplitude for the proton-quark-diquark coupling. The stability condition dictates that the sum of the diquark and parton masses has to be larger than the proton mass to prevent the decay to a free quark and diquark pair. This feature is not apparent from Dyson-Schwinger models of the proton-quark-diquark vertex $[28-30]$, where the diquark mass is of the order $800 \mathrm{MeV}$ and decreases simultaneously with the quark mass at large momentum, but the Euclidean space formulation does not accommodate the decay. Except for this point, the work of these authors can perfectly be reused here as model input.

The vertex functions $g\left(k^{2}\right)$ are known from these fundamental studies to fall with $k^{2}$. One could guess that

$$
g\left(k^{2}\right)=\frac{g}{\left(k^{2}-\Lambda^{2}\right)+\mathrm{i} \epsilon}
$$

for some $\Lambda$ constituent or running quark mass (that we will take fixed here at $0.5 \mathrm{GeV}$, with $g=55$ and $\Lambda=1.6 \mathrm{GeV})$. This is simply suggested by the observation that $T$ is not a truncated amplitude at the quark legs, and therefore it includes their propagators. However, since all the recoil $t$-dependence in the form factor calculation needs to be present in the vertex $g\left(k^{\prime 2}\right)$ and we know by the QCD counting rules that at large momentum transfer $F_{1}(t) \propto$ $t^{-2}$, the simplest workable model is

$$
g\left(k^{2}\right)=\frac{g}{\left(\left(k^{2}-\Lambda^{2}\right)+\mathrm{i} \epsilon\right)^{2}} .
$$

This can be viewed as a derivative with respect to $\Lambda$ of $(31)$; since it can be pulled out of the $k$ integration as a parametric derivative, it does not alter the analytical structure of
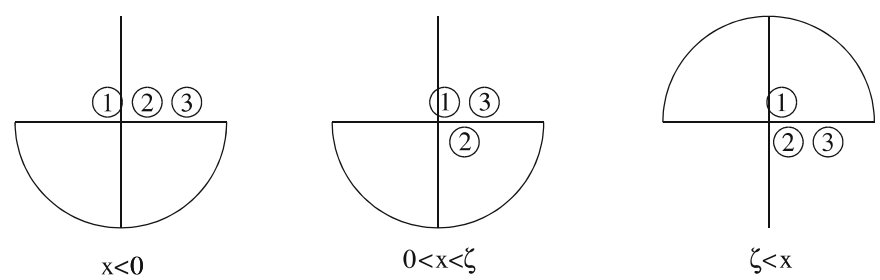

Fig. 2. Integration over the $\kappa^{-}=k^{-} P^{+}$variable in the complex plane for the diquark model. From the figure, $H(x<0)=0$

the DVME amplitude nor the placement of poles of $T$ in the $k^{-}$plane that we are now studying.

Consider, therefore, the model defined by (30) and (32). We employ the variable $\kappa^{-}=P^{+} k^{-}$so that an inverse power of $P^{+}$comes out of the $k^{-}$integral and yields

$H(x, \zeta, t)=\int_{0}^{\infty} \frac{\left|\mathbf{k}_{\perp}\right| \mathrm{d}\left|\mathbf{k}_{\perp}\right|}{(2 \pi)^{4}} \int_{0}^{2 \pi} \mathrm{d} \phi_{\perp} \int_{0}^{\infty} \mathrm{d} \kappa^{-} T\left[s, t, u, k^{2}\right]$,

in place of (29) and with $k^{+}, k^{\prime}$ fixed as above. The partonproton amplitude is at this order an holomorphic function of $\kappa^{-}$and has three poles, whose positions are depicted in Fig. 2. The diquark propagator yields a simple pole denoted as (1) and given by the condition $u-\lambda^{2}+\mathrm{i} \epsilon=0$. The vertex functions yield two double poles, denoted by (2) for $k^{2}-\Lambda^{2}+\mathrm{i} \epsilon=0$ and (3) for $k^{\prime 2}-\Lambda^{2}+\mathrm{i} \epsilon=0$ respectively. These poles are respectively located at

$$
\begin{aligned}
& \kappa_{1}^{-}=-\frac{\lambda^{2}+\mathbf{k}_{\perp}^{2}-M_{N}^{2}(1-x)}{1-x}-\mathrm{i} \epsilon, \\
& \kappa_{2}^{-}=\frac{\Lambda^{2}+\mathbf{k}_{\perp}^{2}+\mathrm{i} \epsilon}{x} \\
& \kappa_{3}^{-}=\frac{\Lambda^{2}+c^{2}+\mathrm{i} \epsilon}{x-\zeta}
\end{aligned}
$$

where we have grouped

$$
\begin{aligned}
c^{2}= & -\left[t+M_{N}^{2}(1-x)+\left(M_{N}^{2}+\boldsymbol{\Delta}_{\perp}^{2}\right)\right. \\
& \left.\times\left(1+\frac{x}{1-\zeta}\right)-\left(\mathbf{k}_{\perp}-\boldsymbol{\Delta}_{\perp}\right)^{2}\right] .
\end{aligned}
$$

The $\kappa^{-}$integral can now be immediately performed picking up the residue of the simple or double pole as in Fig. 3. We find

$$
\begin{aligned}
& H(x \in(0, \zeta), \zeta, t)=g^{2} \Lambda^{8} \frac{1-\zeta / 2}{(2 \pi)^{3}} \int_{0}^{\infty}\left|\mathbf{k}_{\perp}\right| \mathrm{d}\left|\mathbf{k}_{\perp}\right| \\
& \times \int_{0}^{2 \pi} \mathrm{d} \phi_{\perp}\left(\frac{1}{u-\lambda^{2}} \frac{1}{\left(k^{\prime 2}-\Lambda^{2}\right)^{2}}\right)\left(\frac{x-1}{u-\lambda^{2}}+\frac{2(x-\zeta)}{k^{\prime 2}-\Lambda^{2}}\right)
\end{aligned}
$$

evaluated at the pole $k^{-}=k_{2}^{-}$. The second term, being proportional to $x-\zeta$, does not give a contribution to the imaginary part of the electroproduction amplitude at lead- 

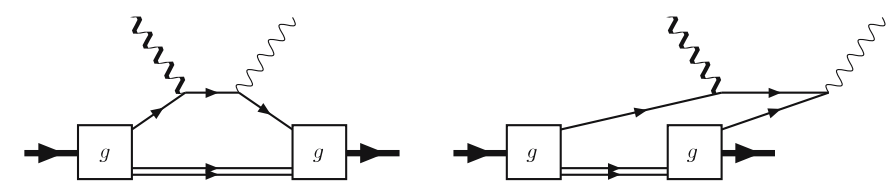

Fig. 3. Two light-cone time-ordered diagrams for the handbag diagram with a covariant parton-proton scattering vertex based on a parton-diquark-proton vertex and second order perturbation theory. Notice that the second diagram, present in a covariant model, gives rise to a non-vanishing $H(x \in(0, \zeta), \zeta, t)$ and to a well-defined imaginary part, by providing a finite part from the left needed for continuity at $x=\zeta$

$\operatorname{ing} Q^{2}$. We also have

$$
\begin{array}{r}
H(x \in(\zeta, 1), \zeta, t)=g^{2} \Lambda^{8} \frac{1-\zeta / 2}{(2 \pi)^{3}} \int_{0}^{\infty}\left|\mathbf{k}_{\perp}\right| \mathrm{d}\left|\mathbf{k}_{\perp}\right| \\
\times \int_{0}^{2 \pi} \mathrm{d} \phi_{\perp}\left(\frac{1}{\left(k^{\prime 2}-\Lambda^{2}\right)^{2}} \frac{1}{\left(k^{2}-\Lambda^{2}\right)^{2}}\right),
\end{array}
$$

which gives a positive definite $H$ function.

\section{Numerical results}

We now provide a numerical computation of the above model in order to illustrate the approach. The $t$-dependence of the $H$ GPD in this quark-diquark model is plotted in Fig. 4. As can be seen, $H$ falls rapidly with $t$ at fixed $\zeta$. The region $\zeta-1<x<0$ cannot be accessed with this "valence" model. However, the fact that the vertex employed is covariant gives a feature absent in the constituent valence light-front quark model, that is, a non-vanishing

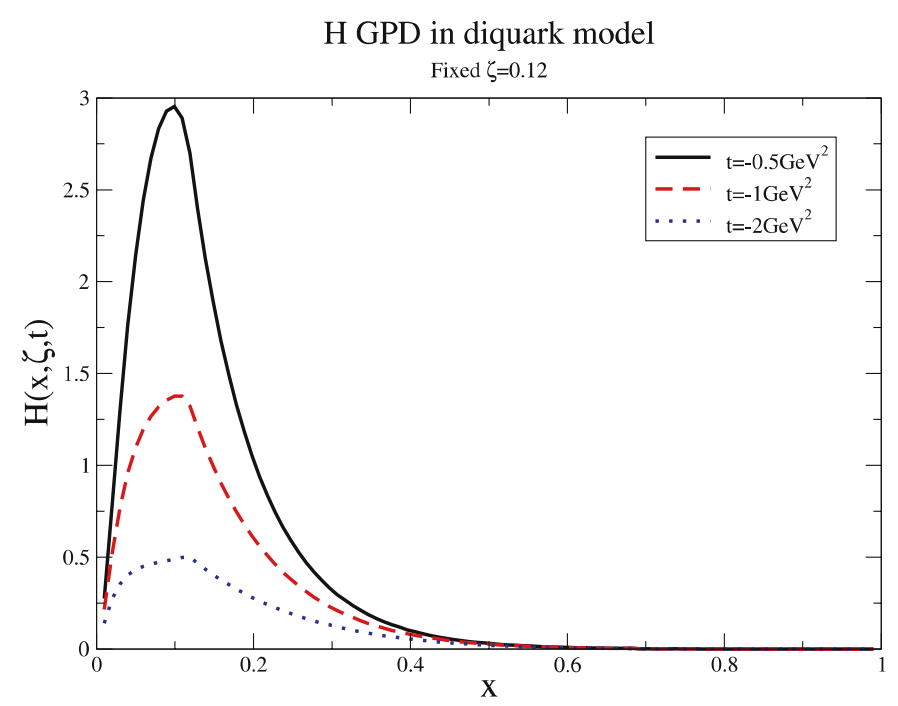

Fig. 4. The $t$-dependence of the $H(x, \zeta, t)$ generalized parton distribution in the perturbative diquark model at fixed $\zeta=0.12$. Since this model does not have a sea wavefunction component, it does not give access to the region $\zeta-1<x<0$. However, it is sufficient for the purpose of illustrating BLM scale fixing via a dispersion relation
$H(x=\zeta, \zeta, t)$ and continuity at $x=\zeta$. This allows for an imaginary part in the electroproduction amplitude in this minimal model. In Fig. 5, we plot the $\zeta$-dependence of $H$. The sharpness of the peak in this graph is controlled by $\lambda$, the diquark mass. The diquark model satisfies the simplifying property

$$
H(x<0, \zeta, t)=0 .
$$

We can then reproduce the result of Belitsky and Muller for the BLM scale for the imaginary part of the amplitude; in our asymmetric frame (23) their result is

$$
\frac{A_{1}}{A_{2}}=\frac{19}{6}+\log \left(\frac{\zeta}{1-\zeta}\right)-\int_{\zeta}^{1} \mathrm{~d} x \frac{\frac{H(x, \zeta, t)}{H(\zeta, \zeta, t)}-1}{x-\zeta} .
$$

In Fig. 6, we display the resulting BLM scale as extracted from the imaginary part of the amplitude. It can be seen that $\mu_{\mathrm{BLM}}^{2} \ll Q^{2}$, indicating that meson electroproduction at practically accessible kinematics measures the coupling constant $\alpha_{s}$ in the infrared regime where theoretical considerations [31] lead us to expect a fixed point. For numerical purposes one can use

$$
\alpha_{s}\left(\mu^{2}\right)=\frac{4 \pi}{\left(9 \log \left(\left(\mu^{2}+M_{0}^{2}\right) / \Lambda^{2}\right)\right)},
$$

with $\Lambda \simeq 0.2-0.21 \mathrm{GeV}$ and $M_{0} \simeq 1-1.1 \mathrm{GeV}$ at the BLM scale in the $\overline{\mathrm{MS}}$ scheme. With this, (17) and (18) can be immediately evaluated. This legacy form of the running coupling has been successfully applied to the prediction of electromagnetic form factors and other quantities (see overview in [32]). Since the BLM scale that we determine is small, our calculations sample mostly $\alpha_{s}(0)$. The value of this fundamental quantity, controlled in (40) by the

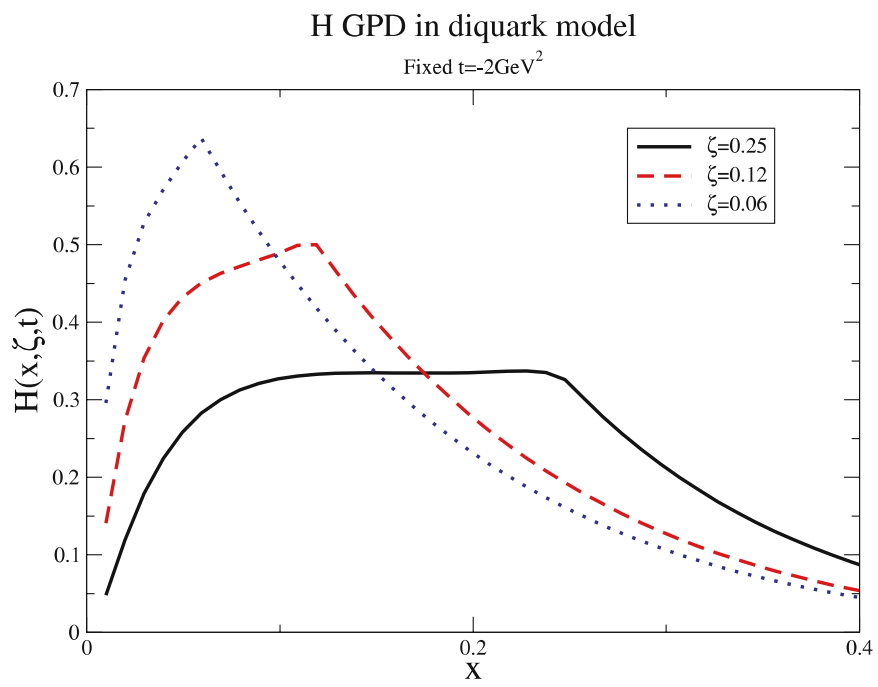

Fig. 5. $\zeta$-dependence of the $H(x, \zeta, t)$ generalized parton distribution in the perturbative diquark model at fixed $t=$ $-2 \mathrm{GeV}^{2}$. Observe that by performing different pole integrals for $x>\zeta$ and $x<\zeta$, obtaining continuity at $x=\zeta$ is a nontrivial test of the model and computations 


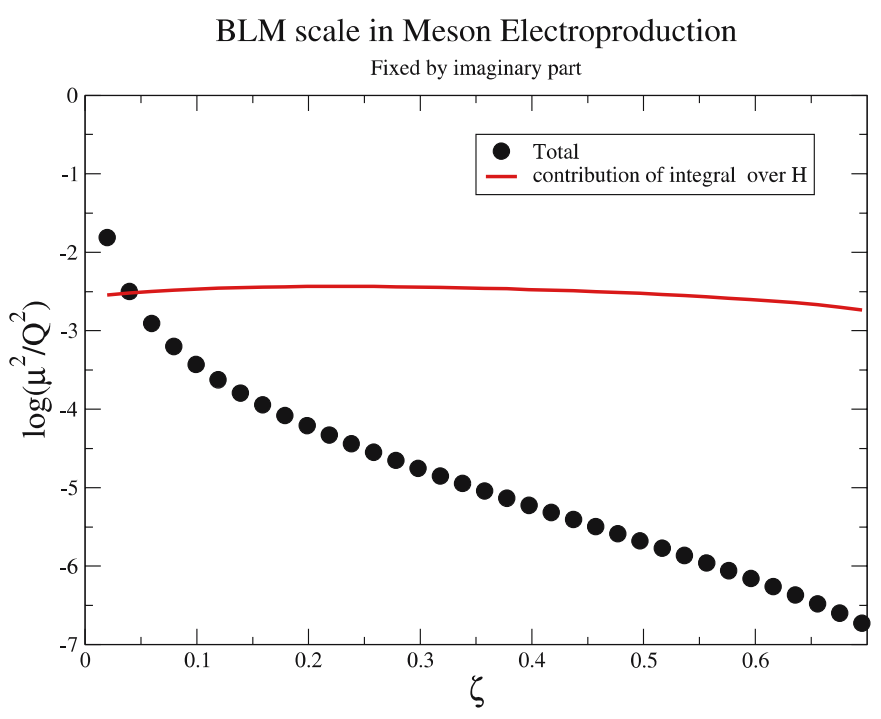

Fig. 6. BLM scale in $\rho_{L}^{0}$ meson electroproduction at $Q^{2}=$ $4 \mathrm{GeV}^{2}$ and $t=-2 \mathrm{GeV}^{2}$. The solid line represents the contribution of the integral in (39)

parameter $M_{0}$, is uncertain. With our parameterization, $\alpha_{s}(0) \simeq 0.9$.

As a final illustration, we give the model's prediction for the differential cross section in (12). The result of the computation is plotted in Fig. 7. Since the perturbative diquark model is only viable at sizeable $t$, we fix $t=-2 \mathrm{GeV}^{2}$. Consequently, the cross section is much smaller than reported by other authors at $t=t_{\text {minimum. The fall-off as }}$ a power law at fixed skewness is built into our formalism can be traced to (14) and (12). Notice that the DVCS cross section is enhanced at high momentum transfer by a factor $\frac{Q^{2}}{f_{\rho}^{2}}$ and eventually crosses over and dominates over $\rho^{0}$ production in spite of the extra $\alpha_{\mathrm{EM}}$ suppression in Compton scattering [23]. For this example, we have chosen kinematics such that $Q^{2} / t$ is 2 or more as a compromise between theory and experimental uncertainties. From the theoretical point of view, one would like to control the factorization corrections increasing this ratio. However a much larger $Q^{2}$ would be difficult for an experiment at an upgraded Jefferson lab or, due to statistical limitations at sizeable $t$, at larger $s$ machines. Note that the largest uncertainty in our work is likely to come from modeling the GPD $H$. The DVME $Q^{-6}$ fall-off is a genuine QCD prediction [18] and is consistent with the experimental result of HERMES [33] at $\sqrt{s}=5.4 \mathrm{GeV}$, which yields an exponent $-5.66(36)$, within one standard deviation. One should notice the subtlety that the HERMES data is quoted at fixed center of mass energy squared $W^{2}=s$ for the photon-nucleon system, whereas the predicted fall-off is at fixed scaling variable, here the skewness $\zeta$. However, since [33] also displays a very weak $W$-dependence, one can slide $s$ in the HERMES data and use our (10) to obtain the same exponent at fixed skewness. The cross sections reported by this experiment, which are dominated by low $t$ Regge processes, cannot be directly compared with our result.

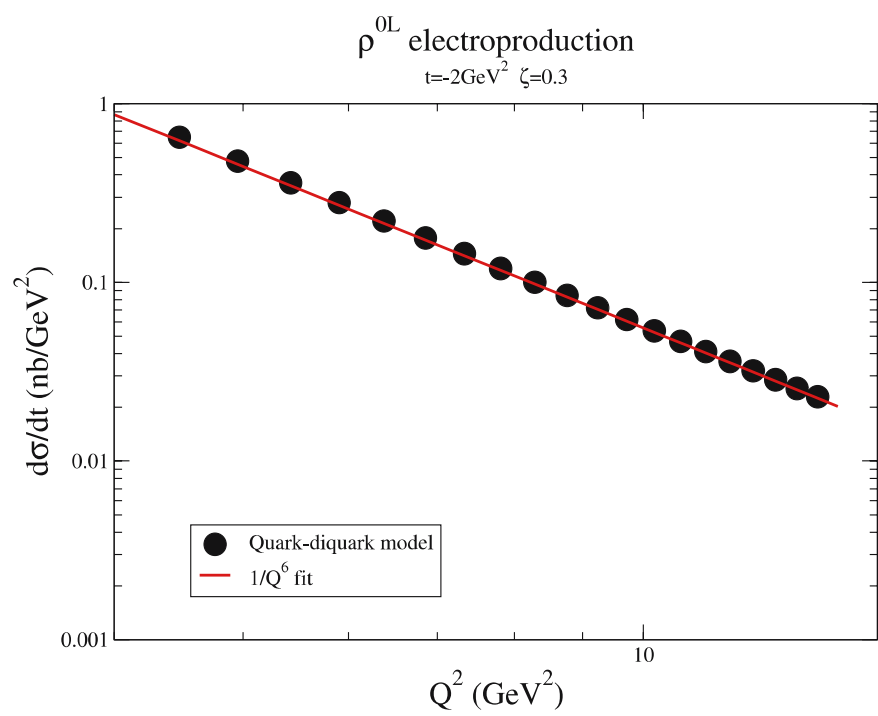

Fig. 7. As an illustration we present the model computation for $\rho_{L}^{0}$ meson electroproduction at $t=-2 \mathrm{GeV}^{2}$ and $\zeta=0.3$. The fall-off with $Q^{6}$ at fixed $\zeta$ follows from (14) and (12)

We look forward to future measurements with a larger $-t$ reach.

\section{Conclusion}

We have shown that there is no a priori difficulty to set the renormalization scales that appear in hard hadronic scattering amplitudes that are genuinely complex. The individual BLM scales for the real and imaginary parts are unambiguous and distinct, and they maintain the analyticity and dispersion relations of the full scattering amplitude. Just as in Abelian theory, the BLM method in QCD fixes the arguments of the running coupling to maintain the correct cut structure of the quark loop vacuum polarization contribution at each flavor threshold. All the non-conformal contributions to the amplitude associated with the running of the gauge coupling are used to set the renormalization scales. The remaining perturbation series is then identical with a conformal theory with zero $\beta$ function.

We have determined the BLM renormalization scales for the imaginary and real parts of the $\gamma^{*} p \rightarrow \rho_{L}^{0} p^{\prime}$ meson electroproduction amplitude, a process of current theoretical and experimental interest. We applied the BLM prescription to the imaginary part and employ a fixed- $t$ dispersion relation to calculate the scale-fixed real part. We have exploited the connection between generalized parton distributions and the parton-proton scattering amplitude in order to obtain an analytic representation of the helicityconserving amplitude $H$ in terms of a simple, yet physically inspired, quark-diquark model of the proton.

It has been conventional to characterize the precision of pQCD predictions by using an arbitrary renormalization scale in the $\overline{\mathrm{MS}}$ scheme, such as $\mu_{R}^{2}=Q^{2}$, and then varying the scale over an arbitrary range, e.g., $Q^{2} / 2<\mu_{R}^{2}<2 Q^{2}$ 
as a means to estimate the convergence of the perturbative series. However, the variation of the renormalization scale can only be relevant for the non-conformal contributions to an observable, not the complete series. In fact, as we have stressed, there is actually no renormalization scale ambiguity since one must set the argument of the running coupling such that amplitudes have the correct analytic cut structure at each quark threshold. This is done correctly in any renormalization scheme by using the BLM method.

Acknowledgements. F.J. Llanes-Estrada warmly thanks the Stanford Linear Accelerator Center's theory group, where this work was carried out, for their hospitality and the Fundacion del Amo-Univ. Complutense fellowship for partial financial support. This work was supported in part by grants FPA 2004-02602, 2005-02327, PR27/05-13955-BSCH (Spain) and US Department of Energy under contract number DE-AC02$76 \mathrm{SF} 00515$.

\section{References}

1. S.J. Brodsky, G.P. Lepage, P.B. Mackenzie, Phys. Rev. D 28, 228 (1983)

2. S.J. Brodsky, C.R. Ji, A. Pang, D.G. Robertson, Phys. Rev. D 57, 245 (1998) [arXiv:hep-ph/9705221]

3. G. Grunberg, A.L. Kataev, Phys. Lett. B 279, 352 (1992)

4. S.J. Brodsky, E. Gardi, G. Grunberg, J. Rathsman, Phys. Rev. D 63, 094017 (2001) [arXiv:hep-ph/0002065]

5. S.J. Brodsky, M.S. Gill, M. Melles, J. Rathsman, Phys. Rev. D 58, 116006 (1998) [arXiv:hep-ph/9801330]

6. E.C.G. Stueckelberg, A. Petermann, Helv. Phys. Acta 26, 499 (1953)

7. S.J. Brodsky, G.T. Gabadadze, A.L. Kataev, H.J. Lu, Phys. Lett. B 372, 133 (1996) [arXiv:hep-ph/9512367]

8. S.J. Brodsky, H.J. Lu, arXiv:hep-ph/9601301

9. S.J. Brodsky, P. Huet, Phys. Lett. B 417, 145 (1998) [arXiv:hep-ph/9707543]

10. M. Gell-Mann, F.E. Low, Phys. Rev. 95, 1300 (1954)

11. J.M. Cornwall, Phys. Rev. D 26, 1453 (1982)

12. T. Appelquist, M. Dine, I.J. Muzinich, Phys. Rev. D 17, 2074 (1978)
13. S.J. Brodsky, M. Melles, J. Rathsman, Phys. Rev. D 60, 096006 (1999) [arXiv:hep-ph/9906324]

14. I.V. Anikin, B. Pire, L. Szymanowski, O.V. Teryaev, S. Wallon, Eur. Phys. J. C 42, 163 (2005) [arXiv:hepph/0411408]; I.V. Anikin, B. Pire, L. Szymanowski, O.V. Teryaev, S. Wallon, Phys. Rev. D 70, 011501 (2004) [arXiv:hep-ph/0401130]

15. G.F. Chew, M.L. Goldberger, F.E. Low, Y. Nambu, Phys. Rev. 106, 1345 (1957)

16. H.J. Bremermann, R. Oehme, J.G. Taylor, Phys. Rev. 109, 2178 (1958)

17. S.J. Brodsky, M. Diehl, D.S. Hwang, Nucl. Phys. B 596, 99 (2001) [arXiv:hep-ph/0009254]

18. S.J. Brodsky, L. Frankfurt, J.F. Gunion, A.H. Mueller, M. Strikman, Phys. Rev. D 50, 3134 (1994) [arXiv:hep$\mathrm{ph} / 9402283]$

19. J.C. Collins, L. Frankfurt, M. Strikman, Phys. Rev. D 56, 2982 (1997) [arXiv:hep-ph/9611433]

20. M. Vanderhaeghen, P.A.M. Guichon, M. Guidal, Phys. Rev. D 60, (1999) [arXiv:hep-ph/9905372]

21. M. Diehl, Phys. Rep. 388, 41 (2003)

22. G.P. Lepage, S.J. Brodsky, Phys. Rev. D 22, 2157 (1980)

23. S.J. Brodsky, F.J. Llanes-Estrada, work in preparation

24. A.V. Belitsky, D. Mueller, Phys. Lett. B 513, 349 (2001) [arXiv:hep-ph/0105046]

25. S.J. Brodsky, F.E. Close, J.F. Gunion, Phys. Rev. D 8, 3678 (1973)

26. P.V. Landshoff, J.C. Polkinghorne, R.D. Short, Nucl. Phys. B 28, 225 (1971)

27. D.D. Coon, J.F. Gunion, J. Tran Thanh Van, R. Blankenbecler, Phys. Rev. D 18, 1451 (1978)

28. P. Maris, arXiv:nucl-th/0412059

29. J.C.R. Bloch, C.D. Roberts, S.M. Schmidt, A. Bender, M.R. Frank, Phys. Rev. C 60, 062201 (1999) [arXiv:nuclth/9907120]

30. M. Oettel, R. Alkofer, L. von Smekal, Eur. Phys. J. A 8, 553 (2000) [arXiv:nucl-th/0006082]

31. R. Alkofer, C.S. Fischer, F.J. Llanes-Estrada, Phys. Lett. B 611, 279 (2005) [arXiv:hep-th/0412330]

32. S.J. Brodsky, T. Huang, G.P. Lepage, SLAC-PUB-2868, Based on lectures given at 9th SLAC Summer Inst. on Particle Physics, Stanford, CA, Jul 27-Aug 7, 1981

33. A. Airapetian et al. [HERMES Collaboration], Eur. Phys. J. C 17, 389 (2000) [arXiv:hep-ex/0004023]; see also the overview in R. Enberg, Nucl. Phys. A 755, 595 (2005) 